\title{
Silicon Photonic Spectrometer for Accurate Peak Detection Using the Vernier Effect and Time-Domain Multiplexing
}

\author{
Alfonso Ruocco, Student Member, IEEE, Dries Van Thourhout, Member, IEEE, \\ and Wim Bogaerts, Member, IEEE, Member, OSA
}

\begin{abstract}
We present a silicon-on-insulator spectrometer for wavelength peak detection operating in a $20-\mathrm{nm}$ wavelength band around $1550 \mathrm{~nm}$ with an average accuracy of $12 \mathrm{pm}$. The silicon photonic integrated circuit occupies a footprint of $250 \times$ $700 \mu \mathrm{m}^{2}$ and is composed of a passive-arrayed waveguide grating (AWG) and an array of thermo-optic Mach-Zehnder interferometer switches. The AWG multiplexes multiple inputs and outputs with a vernier design that shifts the spectral response of the different inputs. In this demonstration, we use five output channels and four input channels, with a 4.0-nm channel spacing and 5.0-nm channel spacing, respectively. The responses to the different inputs are disentangled at the outputs by time-multiplexing the inputs with the switches. We demonstrate a more than tenfold improvement in peak detection accuracy using the Vernier effect, from $\mathrm{nm}$ range using a single input to picometers range using the four inputs.
\end{abstract}

Index Terms-Arrayed waveguide grating, silicon photonics, spectrometer.

\section{INTRODUCTION}

A LARGE class of optical sensors is based on a wavelengthselective device that filters out one or more narrow wavelength peaks, and the response is measured by the spectral shift of this peak. Examples include fiber-bragg gratings [1], but also ring-resonator sensors on chip [2],[3]. While the transducer mechanism is usually straightforward, the difficulty lies in the accurate measurement of the position of the wavelength peak. Usually a bulky spectrometer with movable parts, or an expensive tunable laser is required. Integrated solutions based on passive wavelength filters (e.g. Echelle Gratings [4] or arrayed waveguide gratings (AWG) [5], [6]) often don't offer the required spectral accuracy.

Integrated photonics, and in particular Silicon On Insulator (SOI) technology offers an opportunity for low-cost, mass

Manuscript received March 16, 2014; revised July 11, 2014; accepted August 5, 2014. Date of publication August 7, 2014; date of current version August 27, 2014. This work was supported by the European FP7-SmartFiber Project.

A. Ruocco and D. V. Thourhout are with the Photonics Research Group, Department of Information Technology, Center of Nano-and Biophotonics, Ghent University-IMEC, 9000 Gent, Belgium (e-mail: alfonso.ruocco@ intec.ugent.be; driesvt@intec.ugent.be).

W. Bogaerts is with the Photonics Research Group, Department of Information Technology, Center of Nano-and Biophotonics, Ghent University-IMEC, 9000 Gent, Belgium, and also with the Luceda Photonics, 9200 Dendermonde, Belgium (e-mail: wim.bogaerts@intec.ugent.be).

Color versions of one or more of the figures in this paper are available online at http://ieeexplore.ieee.org.

Digital Object Identifier 10.1109/JLT.2014.2346585

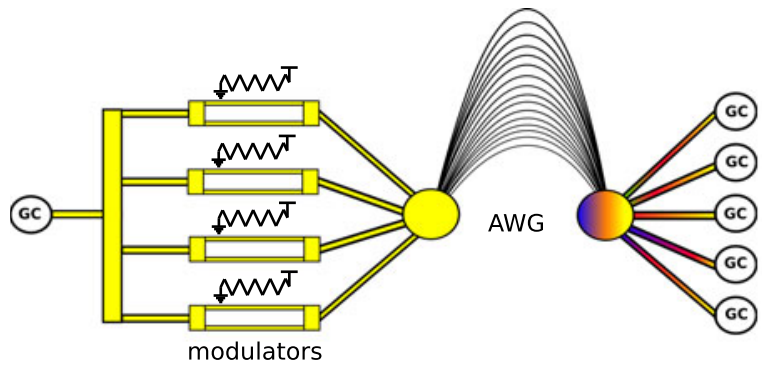

(a)

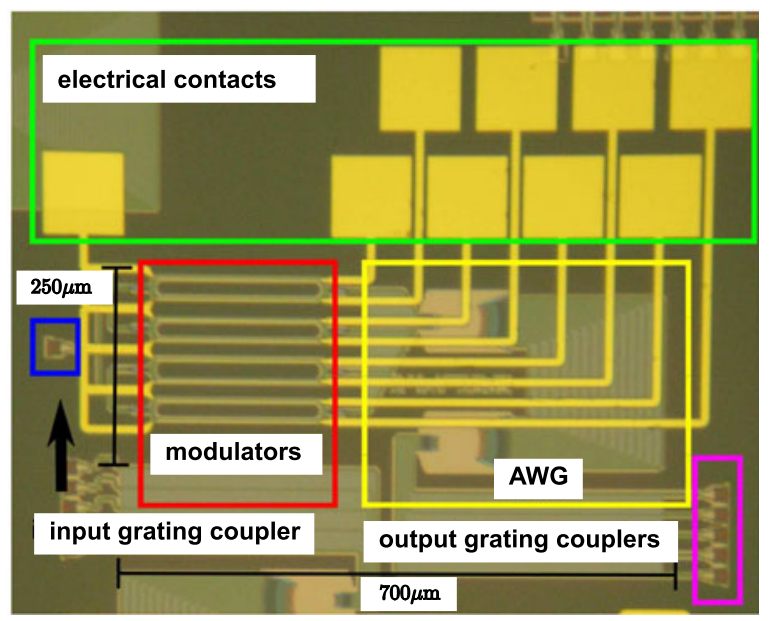

(b)

Fig. 1. Spectrometer schematic and photo. (a) Device schematic (b) Device microscope image.

manufactured optical systems, including spectrometers [7] and wavelength sensor interrogators [8]-[10]. On the other hand their performance is limited by the technology. Especially a high wavelength accuracy is difficult to achieve, as the channel spacing cannot be scaled down indefinitely. In this work we use active multiplexing to improve wavelength accuracy of an onchip passive wavelength detector. The basic filter component we use is an AWG [5]. This device is a widely used component in Wavelength Division Multiplexing (WDM) systems, and its use has expanded in other fields like signal processing, sensing and spectroscopy. SOI-based AWG are very compact, and becoming increasingly efficient [11]. An AWG wavelength demultiplexer with a single input and multiple outputs can be used as a peakdetection spectrometer: by measuring the power in the different 
output channels the location of a wavelength peak can be determined with such method the raw resolution is approximately the output channel spacing. This can even be done with a higher accuracy than the AWG channel spacing by weighing power ratios between different outputs [12]. Still, the final accuracy of the device will be limited by the unsuitable part of each wavelength channel, close to the zero derivative points. In this work we apply Vernier scaling to the AWG to significantly boost the accuracy of the peak detection: we add additional inputs to the passive device as represented in Fig. 1(a), while Fig. 1(b) shows a microscope image of the entire device. We then send the input signal through the AWG and register the response signal in the output channels of all inputs combined. To disentangle the response coming from the different inputs, we 'label' each input signal using a modulator. That way we can unravel the input signals using signal processing techniques. The modulators are symmetric MZI interferometers with a thermo-optic phase shifter in one arm. The length of the MZI is overdimensioned to obtain a $\pi$ phase shift with a sufficiently low drive current. Using the vernier effect, we manage to increase the accuracy of the device. In this case, using 4 input channels and 5 output channels, we obtain a tenfold improvement. In this paper, we use the most simple multiplexing technique: sequential timedomain multiplexing (TDM), effectively using the thermo-optic modulator as a simple on-off switch: at any given time, only one input channel is switched on. Alternatives to this modulation scheme could be Frequency Division Multiplexing (FDM) or Code Division Multiple Access (CDMA), where the responses can be disentangled using digital signal processing. The paper is structured as follows: first, we describe the circuit in more detail in section II. In Section III we describe the design and simulation of the device. Section IV discusses the measurement procedure, and finally, we present the experimental results in Section V.

\section{DESCRIPTION OF THE CiRCUIT}

Our device consists of a silicon photonic integrated circuit. The circuit contains a single AWG, and four MZI switches, one for each input channel of the AWG. The optical circuits is realized in the passive SOI technology platform of IMEC [13], in a CMOS-compatible pilot line. The $200 \mathrm{~mm}$ SOI wafer substrates have a $2 \mu \mathrm{m}$ buried oxide, and a $220 \mathrm{~nm}$ silicon layer which is used for the waveguide patterning. On top, an additional $2 \mu \mathrm{m}$ of oxide cladding is deposited. A curved grating coupler [14] is used for the optical inputs and outputs. The input light is divided into four branches. Each branch has its own MZI switch, with one input and output, using a compact $1 \times 2$ Multi-mode interferometer as a splitter and combiner. From the switch, the light is injected into the different inputs of the AWG. On the output side of the AWG, an array of five grating couplers is used to extract the light from the chip.

\section{A. $A W G$}

The AWG consists of two Free Propagation Region (FPRs) and an array of waveguides, with an equal path length difference between them. The longer the path length difference, the smaller

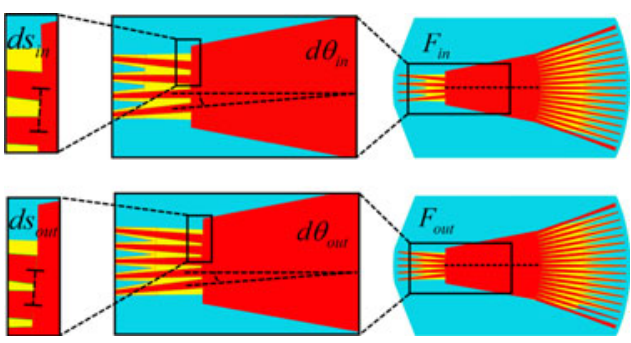

Fig. 2. In and out star couplers: design difference.

the free spectral range (FSR) of the AWG [15]. For a given number of waveguides in the array, a smaller FSR means a higher spectral resolution of the passive spectrometer and a better accuracy in wavelength detection. However, this is limited by the increase in phase errors induced by longer arms. With the current state of technology, this limits the usable channel spacing of these silicon AWGs to around $1 \mathrm{~nm}$ or higher. In our device, we use a channel spacing of $4 \mathrm{~nm}$ for the input. The number of arms in our device is lower than in the case of WDM communication. For a communication mux/demux design, the standard number of waveguides in the array is approximately four times the number of wavelength channels: a number that ensures sharp roll-off and sufficiently low crosstalk levels. We fixed the number of waveguides to three times the number of the output channels. The lower number of arms reduces the crosstalk floor due to random phase errors, but also widens the channel (less steep roll-off) en thus enlarges the overlap between neighboring channels. This design approach is useful for finding wavelength peaks that are located in the middle between two channels. Therefore in our case, we prefer a lower number of arms. The star couplers are laid out in a Rowland mounting [16], and the transition between the waveguides and the FPR is done using a deep and shallow etch, to reduce unwanted reflections [6]. The output waveguides are positioned such that the light of the required wavelength is focused on the respective physical aperture. Fig. 2 shows how the inputs and outputs are configured in the two star couplers. If we consider the spectral position of the AWG channels, the different combinations of $M$ inputs and $N$ outputs can be expressed as an $M \times N$ matrix (1). The element in eq. (1) represent the central wavelength of the in/out channels combination. Every row and column represents an input and an output, and the matrix elements are the peak wavelengths in the response from a given input to a given output. For a wavelength (de)multiplexer, this matrix would be a $1 \times N$ matrix. For a wavelength router, it would be an $N \times N$ matrix, where every element satisfies the eq. (2).

$$
\begin{aligned}
P_{\text {pos }} & =\left(\begin{array}{ccccc}
\cdot & & & & \cdot \\
\cdot & \lambda_{n-1, m-1} & \lambda_{n-1, m} & \lambda_{n-1, m+1} & \cdot \\
\cdot & \lambda_{n, m-1} & \lambda_{n, m} & \lambda_{n, m+1} & \cdot \\
\cdot & \lambda_{n+1, m-1} & \lambda_{n+1, m} & \lambda_{n+1, m+1} & \cdot \\
\cdot & \cdot & \cdot & \cdot & \cdot
\end{array}\right) \\
\lambda_{i, j} & =\lambda_{(i, j) \pm 1} .
\end{aligned}
$$


In this case, the channel spacing at the inputs is equal to the channel spacing at the output. This translates in a physical spacing of the output waveguides which is the same for input and output star coupler. But this configuration is not very useful for a spectrometer: even though there are $N \times N$ elements in the matrix, the transmission does not contain any additional spectral information compared to $1 \times N$ device. However, when we use a different number of $M$ input channels and $N$ output channels, where the channel spacing at the input is different from that at the output, we can arrange it in such a way that all the central wavelengths of the different input-output combinations are different. At that point, much more spectral information can be obtained if we can read out all these matrix elements individually. We can choose the input channels such that they are evenly spaced, and with the same FSR as the output channels. As the FSR remains constant, the device has a cyclic behavior, similar to a wavelength router. The ratio between $M$ and $N$ is called the Vernier factor $n_{\text {vernier }}$ eq. (3). The relation between the input and output channel spacing is obtained through the eq. (4):

$$
\frac{M}{N}=n_{\text {vernier }}
$$

A vernier factor can be used to maximize the spectral information that can be extracted from an $N$-channel device. Fixing the Vernier factor $n_{\text {vernier }}$ (as ratio of integer numbers) and the number of output channels $N$, the number of input channels $M$ and the input channels spacing $I_{\mathrm{chsp}}$ can be calculated:

$$
I_{\text {chsp }}=\frac{O_{\text {chsp }}}{n_{\text {vernier }}} .
$$

\section{B. Mach-Zehnder Switch Design}

When using multiple inputs in an AWG at the same time, the intensities will add up incoherently in the photodetectors, and the additional spectral information will be lost. Therefore, a mechanism is needed to disentangle the contributions from the different inputs. This can be done by multiplexing: by electronically 'labeling' the different input signals, we can separate them with signal processing on the photodetector electrical output. The simplest form of multiplexing we can use here is TDM. By selectively switching on one input at a time, we can sequentially read out the signals from the different inputs. For this, we need electro-optical switches on each input. We implement those using a thermally actuated symmetric MZI. Each MZI is composed of two $3 \mathrm{~dB}$ splitter/combiners with a $100 \mu \mathrm{m}$ long waveguide in between. To implement the thermo-optic switching, we added metal heaters to the passive silicon chip. On the SOI circuit with oxide cladding, layers of metal are deposited. The actual heater is a strip of titanium of about $100 \mathrm{~nm}$ thick and 2 um wide. On top of the titanium, a layer of gold of few hundred $\mathrm{nm}$ is deposited to reduce the resistance of wires and pads; on the heater itself no gold is deposited. All the heaters have a common ground. The MZI is symmetric: both arms have the same length, chosen to guarantee a safe range of thermal driving power of the metallic heaters. Under these conditions, the passive response of each modulator is an all-pass device: all the inputs are in the ON state simultaneously. We use the thermo-optic effect [17], [18] to induce a $\pi$ phase change in one of the arms, to drive the individual MZIs into destructive interference for the OFF state. The drawback of the thermo-optic effect is the low driving speed which is limited to few hundred $\mu s$. But compared to plasma-dispersion silicon modulators, the thermo-optic devices have a low insertion loss.

\section{Use of the Device for Peak Detection}

The position of the peak can be estimated from the ratio of the channel outputs as in [8]. The limitation of accuracy and range for such a device is similar to the trade-off between channel spacing, number of channels and FSR of WDM demultiplexer components. Using other configurations with multiple AWGs, these limitations can be pushed back, but the use of multiple filter devices can suffer from non-uniformities introduced by the fabrication process. Especially in silicon, the separate AWGs can have a different fabrication-induced wavelength shift. The wavelength range of the device is still limited by the FSR of the AWG, in this case $20 \mathrm{~nm}$. In the following algorithm, we assume the wavelength response of the channels to be gaussian, but the reasoning holds for other spectral response shapes. Let us call the photodetector signal $S_{n, m}$ the integral (over all wavelengths) of the light that passes from input $m$ to output $n$ (assuming ideal quantum efficiency of the photodetector), and an input signal that consists of a single wavelength peak $\delta(\lambda)$.

$$
S_{n, m}=\int_{\lambda} G_{n, m}(\lambda) \delta_{\text {peak }}(\lambda) d \lambda
$$

Where the kernel function $G_{n, m}(\lambda)$ represents the pseudogaussian response curve of each wavelength channel of the AWG. Of course, the actual signal $S_{n}$ in the photodetector of output $n$ consists of the multiplexed contributions from all $M$ inputs:

$$
S_{n}=\sum_{n=0}^{M} \int_{\lambda} \hat{G}_{n}(\lambda, t) \delta_{\text {peak }}(\lambda) d \lambda .
$$

We can extract the useful signals $S_{n, m}$ from the measured signals $S_{n}$ by electronically disentangling them. When using TDM, $S_{n}$ is a time-dependent signal, and so is the kernel function $\hat{G}_{n}(\lambda, t)$. At any given time, the measured value of $S_{n}$ corresponds to one of the $M$ signals $S_{n, m}$. For other multiplexing methods (FDM or CDMA) the signals can be disentangled in a similar way. This way, we extract $M \times N$ signals from the $N$ photodetectors. This technique can now be used to estimate the wavelength position of the peak $\delta(\lambda)$. In a single input AWG wavelength detector, measurable signals for peak revelation are usually collected from only two neighboring channels. Using the Vernier scaling, we have more densely spaced spectral channels. A Center of Gravity (CoG) [19], [20] algorithm can be used to increase the accuracy of the device. An equivalent mass value is assigned to each channel intensity: the equivalent mass value is higher when the position of the peak is closer to the center of the channel. Only the signal beyond a certain threshold are taken into account: this selection is applied to filter out noise. This approach gives an equivalence between the classic use of $\mathrm{CoG}$ 
and the wavelength detection used here. Considering the disentangled signals $S_{n, m}$ coming from the photodiodes, the CoG algorithm can be expressed as eq. (7). The average accuracy of the spectrometer is evaluated according to the eq. (8).

$$
\begin{aligned}
\lambda_{\text {meas }} & =\frac{1}{\sum_{m=1}^{M} S_{n, m}} \sum_{m=1}^{M} \sum_{n=1}^{N} \lambda_{n, m} S_{n, m} \\
S_{\text {acc }} & =\frac{\sum_{n=1}^{M}\left(\sqrt{\left.\lambda_{\text {in }}-\lambda_{\text {meas }}\right)^{2}}\right.}{M} \\
\lambda_{\text {calibrated }} & =\lambda_{\text {meas }}-\left(\sum_{k=0}^{K} A_{k} e^{j 2 \pi k \lambda_{\text {meas }}}\right)^{-1} .
\end{aligned}
$$

In eq. (7) $\lambda_{n, m}$ is the peak position of the channel of interest and $S_{n, m}$ in eq. (5) the signal proportional to the photocurrent collected by the photodiode $n$. With this procedure the only calibration data used is a matrix containing the positions of the maxima belonging to each input/output channel combination of the AWG, in this particular case 20 elements. The characteristic error curve of the spectrometer shows that the CoG method generates a sawtooth like error behavior (see Section IV). The stepping is due to each introduction of a new AWG channel and the removal of the previous one in the wavelength sweep. The channel introduction and removal is due to the threshold. The linear characteristic is due to the symmetric wavelength density of channels. Such stepped slope corresponds to a linear behavior in the $\mathrm{CoG}$ algorithm. The slope mismatch is due to thresholding of the accepted signals and AWG non-idealities. If the function is injective, a complete calibration can be performed to take into account the intrinsic error of the device. With this approach only the random part affects the measurement. Because of its sawtooth-like characteristic, the systematic error using simple $\mathrm{CoG}$ algorithm can be analyzed and modeled. The resulting components of the Fourier series relative to the systematic error are used for a fine calibration of the device according to the eq. (9). This calibration leads to a "weight" CoG algorithm. The wavelength peak we want to detect is assumed to be ideal as well. This is a reasonable assumption as long as the $3 \mathrm{~dB}$ bandwidth of the wavelength peak is at least $10 \times$ narrower than the $3 \mathrm{~dB}$ bandwidth of the AWG channel. The CoG algorithm can't be reliably used in a configuration with single-input AWG because each peak is collected with significant power by only one channel. In our configuration we overcome this limitation because in every part of the spectrum there are multiple channels active. The wavelength positions for the calibration data are obtained by full characterization of the device. The approach used is valid only if the temperature is kept constant: with different temperatures the position of the channels and consequently the sawtooth like curve change according to the new conditions induced by the new thermal equilibrium, indeed in all of the used expressions temperature is a variable (the wavelength shift in function of the temperature is around $\left.0.1 \mathrm{~nm} /{ }^{\circ} \mathrm{C}\right)$. Since the temperature is kept constant at $25^{\circ} \mathrm{C}\left(<0.5^{\circ} \mathrm{C}\right)$ using a Thermoelectric Cooler, it is not considered further in the analysis.

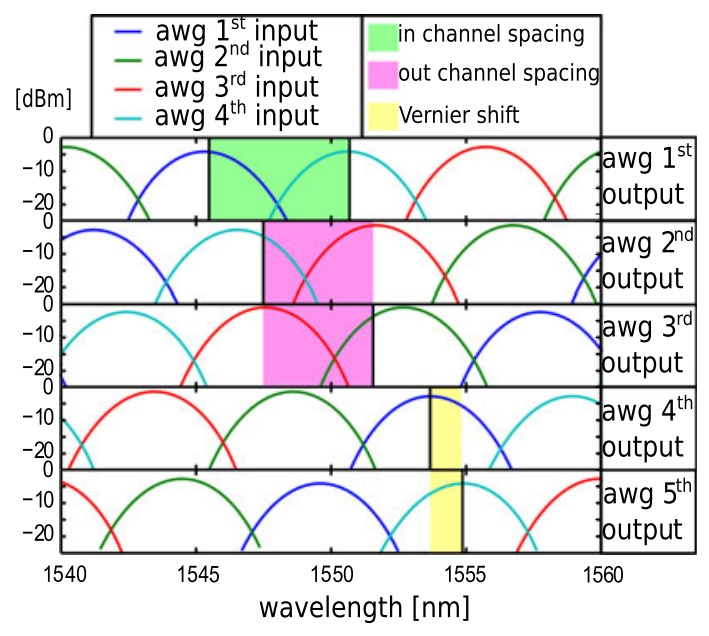

Fig. 3. Spectral simulation of the device.

\section{Simulation OF THE PIC}

We simulate our device in the Caphe circuit simulator [21]. For this, a circuit model for the individual building blocks is needed. For the waveguides (with heaters), splitters and combiners, a simple S-matrix model is defined. For the AWG, the model is calculated by breaking up the device into its separate parts and simulating those parts independently [22]. The coordination of these simulations is done with the IPKISS design framework [23]. The resulting model is then used in the Caphe circuit simulator. Fig. 3 shows the results of the simulation of our photonic integrated circuit: each plot shows the $M$ signals $S_{n, m}$ coming from all the $M$ inputs, collected and de-labelled on one of the $N$ outputs. In the Fig. 3 the input channel spacing, the output channel spacing and the Vernier shifting of the relative channel position are also indicated. The simulated MZI switches include losses and 20-30 dB ON/OFF suppression ratio.

\section{Characterization of the Device}

\section{A. Mach-Zehnder Switches Characterization with Setup 1}

The active and passive parts of the circuit are characterized separately. We characterized the four thermo-optic switches to determine the voltage required for full ON/OFF switching. The OFF Voltage corresponds to the first minimum in Fig. 4, marked with $\times$. In these points, with a single input channel in the OFF state, we see a power drop of approximately $25 \%(-1.5 \mathrm{~dB})$. However, we found some non-uniformity in the switching voltages for the four MZMs, listed in Table I. The used setup is schematically represented in Fig. 6. For this characterization we use a broadband Superluminescent LED (SLED) centered at $1550 \mathrm{~nm}$, and the output light is collected from a single output grating coupler through a fiber vertical coupling and redirected to an OSA. The OSA is used as a power meter of the integrated power in Fig. 4 and as a spectrum analyzer in Fig. 5, measuring the spectral power density. Fig. 5 shows the spectrum with the input channels switched alternatively off using the voltage values obtained in Fig. 4. We see a suppression ratio 


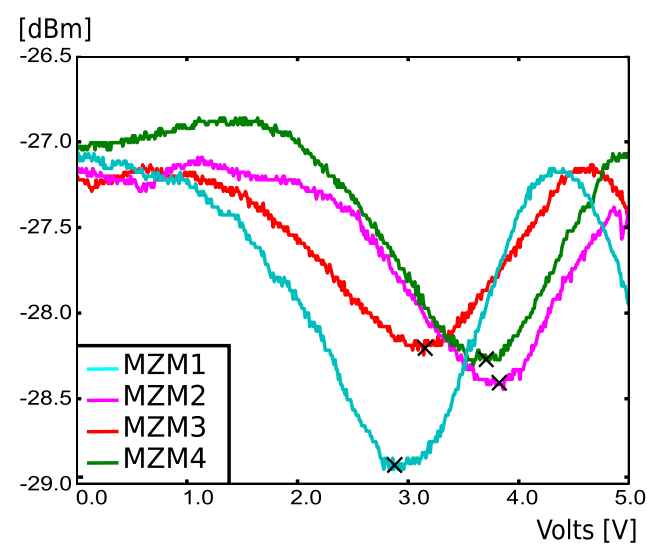

Fig. 4. Integral optical output power sweeping the MZMs voltage (setup 1 with OSA as powermeter).

TABLE I

MZMS CHARACTERIZATION RESULTS

\begin{tabular}{lcccc}
\hline \hline & Input 1 & Input 2 & Input 3 & Input 4 \\
\hline Heater driving voltage & 2.9 & 3.9 & 3.2 & 3.8 \\
ON/OFF suppression ratio & $13 \mathrm{~dB}$ & $18 \mathrm{~dB}$ & $16 \mathrm{~dB}$ & $18 \mathrm{~dB}$ \\
\hline \hline
\end{tabular}

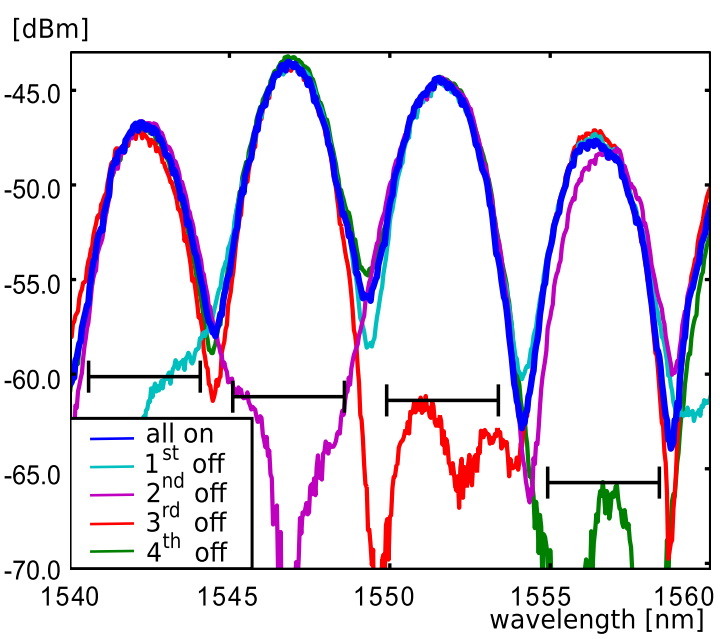

Fig. 5. Spectral channels suppression ratio (setup 1).

of 13-18 dB. The spectra in Fig. 5 also explain the nonuniformity in Fig. 4: As the AWG has a cyclic behavior, for some channels an additional AWG order is captured within the bandwidth of the SLED+grating coupler. The presence or absence of this additional spectral lobe will give a relatively larger or smaller change in the total power when that input is switched on or off. To eliminate the effect of inter-channel crosstalk, we apply a threshold filter before we use the CoG method: we use a threshold of $10 \mathrm{~dB}$, which is above the worst extinction ratio of the switches $(-13 \mathrm{~dB})$. By discarding all measurements below the threshold, non-idealities introduces by the switches do not affect the measurement. If for some reason some unwanted signal is higher than the threshold, its contribution will create

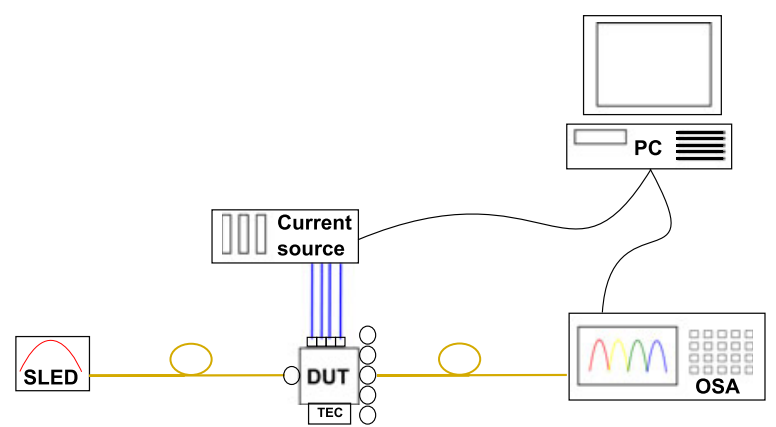

Fig. 6. Setup 1: MZI calibration.

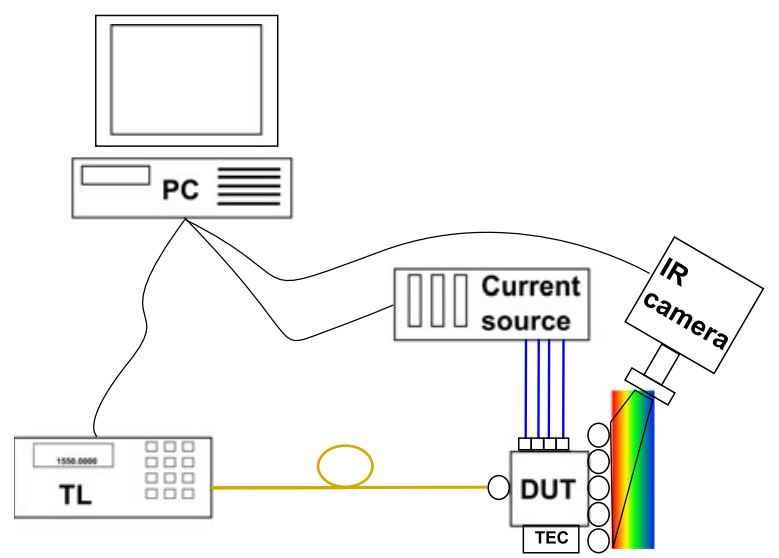

Fig. 7. Setup 2: device calibration and measurements.

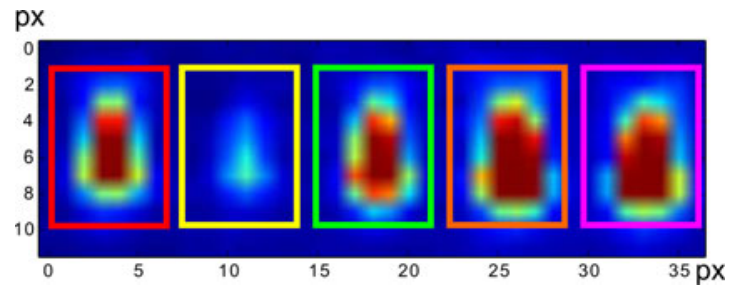

Fig. 8. Captured camera frame (setup 2).

an equivalent mass in the $\mathrm{CoG}$ in a wrong position (wavelength domain) shifting the estimated wavelength away form the actual position.

\section{B. Device Characterization with Setup 2}

For the full characterization of the spectrometer, we use a combination of optical and electrical instruments and the implemented setup in represented in Fig. 7. The array of thermo-optic switches is driven with four independent voltage sources. All grounds are connected to the common ground on our chip. The optical source used is a tunable laser, which can be considered the equivalent of a wavelength peak to be detected. The signals from the output grating couplers are read simultaneously with an infrared (IR) camera. In one camera frame, we can monitor the intensity in different areas corresponding to the different grating couplers. Fig. 8 shows a captured frame. The rectangles 


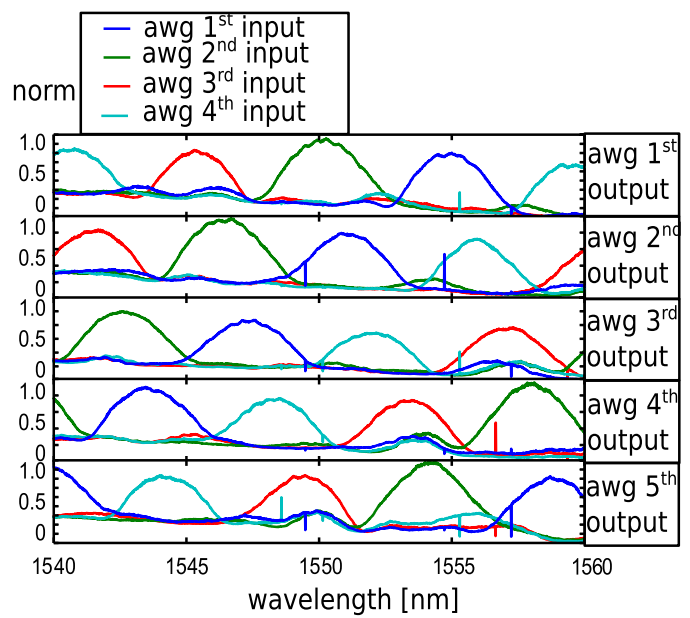

Fig. 9. Spectral measurements of the device (setup 2).

in it are the light spots coming out of the grating couplers. We perform an integration operation inside the selected areas, and thus we get a readout proportional to the power coming out of the grating couplers. We characterize the device using a time division multiplexing scheme on the switches: the MZIs are switched $\mathrm{ON}$ one at the time. Under this condition only one of the inputs is actually optically connected to the AWG. We then perform a wavelength sweep with the laser and collect the IR images at the output grating coupler for each wavelength and input driving combination. With a camera read-out, we can collect the signals from all outputs simultaneously, instead of using a single fiber to sequentially read out the $N$ outputs. Alternatively, we could have designed the chip for read-out with a fiber array, or integrated multiple detectors on chip. Fig. 9 shows the result of the TDM measurement with all the input-to-output spectra disentangled. This corresponds well with the simulation result in Fig. 3. Due to deviations in the SOI fabrication process, the spectrum of the fabricated device is somewhat shifted compared to the design specifications. However, because the response is cyclic, the device can be calibrated in advance.

\section{EXPERIMENTAL RESULTS}

From the measured spectra we can now estimate the position of the wavelength using the procedure described earlier. The resulting measured wavelength is plotted in Fig. 10(a) against the actual input wavelength as set in the tunable laser. The ideal response of our device would be a straight line where the measured wavelength is equal to the input wavelength. We see that the experiment comes close to the straight line. Fig. 10(b) shows the difference between the measurement value and the straight line, which gives us the accuracy of the wavelength detection according to eq. (8). The raw measured accuracy of the CoG method is of the order of $\pm 0.5 \mathrm{~nm}$, while average accuracy is $180 \mathrm{pm}$ and is evaluated according to eq. (8). If the sawtoothlike behavior of the characteristic curve is taken into account (cf. Fig. 10(a) (zoom detail) and eq. (9)) the accuracy of the device is of the order of $\pm 0.2 \mathrm{~nm}$, but the average accuracy is improved to $12 \mathrm{pm}$. The estimated dynamic range is of about

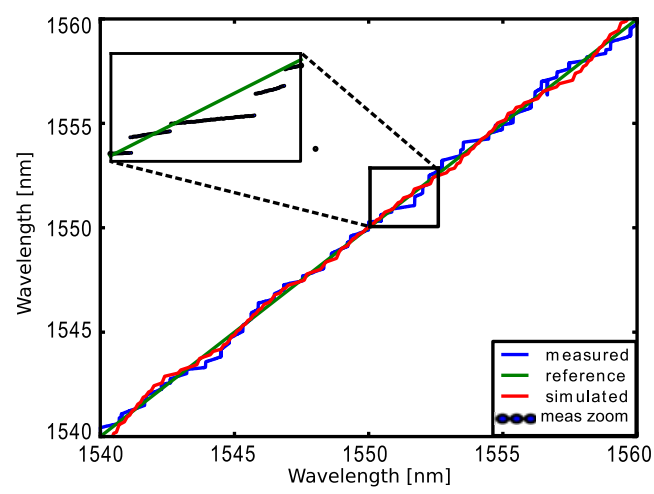

(a)

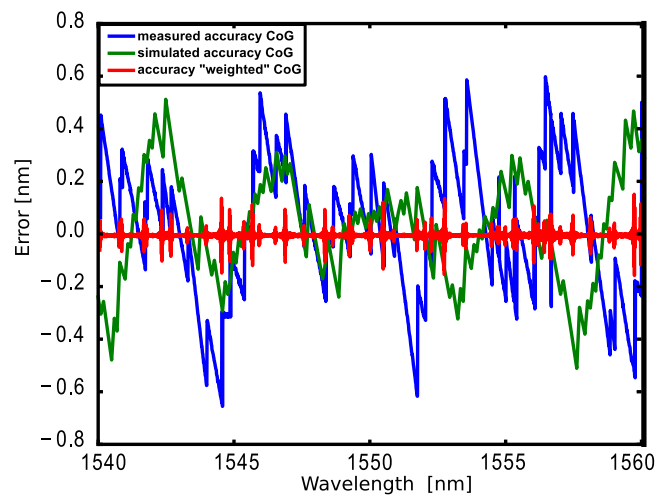

(b)

Fig. 10. Device characteristic curves. (a) Absolute measured wavelength (b) Device local accuracy.

$20 \mathrm{db}$, this number is obtained considering the IR camera saturation level (between 10 and $13 \mathrm{dBm}$ ) and the threshold used for data selection (between -10 and $-12 \mathrm{dBm}$ ). Fig. 10(a) (red curve), shows the local error using the weight" $\mathrm{CoG}$ and points out that the error is mainly caused by fast derivatives in the characteristic curve (cf. error zoom in Fig. 10(b)), in other areas the average error is in the order of pm. More advanced fitting and calibration techniques can further improve the performance. Since the present work is a proof of concept, both peak accuracy and average accuracy are mentioned. This because the spikes in the red curve of Fig. 10(b) can be eliminated.

\section{CONCLUSIONS}

In this paper we demonstrated a novel configuration of an active AWG-based spectrometers for peak detection implemented in silicon photonics. The passive integrated circuit was simulated, fabricated and characterized. For the active control we used thermo-optic switching. By using the Vernier effect and time-domain multiplexed multiple inputs in our AWG, we can improve the peak detection accuracy by a factor of 10 compared to single input devices and 100 if fine calibration procedure are performed. Moreover such configuration offers more flexibility of use, indeed the PIC developed is able to detect peaks over its entire FSR with no unusable windows. The experimental performance demonstrated an average accuracy of $180 \mathrm{pm}$ with 
standard CoG algorithm and $12 \mathrm{pm}$ with the "weighed" CoG algorithm, across the FSR of the WDM device $(20 \mathrm{~nm})$.

\section{REFERENCES}

[1] P. Niewczas, A. Willshire, L. Dziuda, and J. McDonald, "Performance analysis of the fiber bragg grating interrogation system based on an arrayed waveguide grating," IEEE Trans. Instrum. Meas., vol. 53, no. 4, pp. 1192-1196, Aug. 2004.

[2] T. Claes, W. Bogaerts, and P. Bienstman, "Vernier-cascade label-free biosensor with integrated arrayed waveguide grating for wavelength interrogation with low-cost broadband source," Opt. Lett., vol. 36, no. 17, pp. 3320-3322, Sep. 2011

[3] K. D. Vos, J. G. Molera, T. Claes, Y. D. Koninck, S. Popelka, E. Schacht, R. Baets, and P. Bienstman, "Multiplexed antibody detection with an array of silicon-on-insulator microring resonators," IEEE Photon. J., vol. 1, no. 4, pp. 225-235, Oct. 2009.

[4] J. Brouckaert, G. Roelkens, S. Selvaraja, W. Bogaerts, P. Dumon, S. Verstuyft, Z. Yu, D. V. Thourhout, and R. Baets, "Miniature integrated spectrometer fabricated on a silicon-on-insulator substrate," in Proc. 21 st Annu. Meeting IEEE Lasers Electro-Opt. Soc., 2008, pp. 55-56.

[5] M. K. Smit and C. van Dam, "Phasar-based wdm-devices: Principles, design and applications," IEEE J. Sel. Topics Quantum Electron., vol. 2, no. 2, pp. 236-250, Jun. 1996.

[6] W. Bogaerts, S. Selvaraja, P. Dumon, J. Brouckaert, K. D. Vos, D. V. Thourhout, and R. Baets, "Silicon-on-insulator spectral filters fabricated with CMOS technology," IEEE J. Sel. Topics Quantum Electron., vol. 16, no. 1, pp. 33-44, Feb.2010.

[7] E. Ryckeboer, A. Gassenq, M. Muneeb, N. Hattasan, S. Pathak, L. Cerutti, J. B. Rodriguez, E. Tourni, W. Bogaerts, R. Baets, and G. Roelkens, "Silicon-on-insulator spectrometers with integrated gainassb photodiodes for wide-band spectroscopy from 1510 to 2300 nm," Opt. Exp., vol. 21 no. 5, pp. 6101-6108, Mar. 2013.

[8] Y. Sano and T. Yoshino, "Fast optical wavelength interrogator employing arrayed waveguide grating for distributed fiber bragg grating sensors," J. Lightw. Technol., vol. 21, no. 1, pp. 132-139, Jan. 2003.

[9] G. Z. Xiao, P. Zhao, F. G. Sun, Z. G. Lu, Z. Zhang, and C. P. Grover, "Interrogating fiber bragg grating sensors by thermally scanning a demultiplexer based on arrayed waveguide gratings," Opt. Lett., vol. 29, no. 19, pp. 2222-2224, Oct. 2004.

[10] H. Lia, Y. Lia, E. Lib, X. Donga, Y. Baia, Y. Liua, and W. Zhoua, "Temperature-insensitive arrayed waveguide grating demodulation technique for fiber bragg grating sensors," Opt. Laser Technol., vol. 51, pp. 77-81, Oct. 2013.

[11] S. Pathak, M. Vanslembrouck, P. Dumon, D. V. Thourhout, and W. Bogaerts, "Effect of mask grid on SOI arrayed waveguide grating performance," in Proc. 10th Int. Conf. Group IV Photon., 2013, pp. 31-32.

[12] X. G. H. H. Su, "A novel fiber bragg grating interrogating sensor system based on AWG demultiplexing," Opt. Commun., vol. 275, no. 1, pp. 196-200, Jul. 2007.

[13] S. Selvaraja, W. Bogaerts, P. Dumon, D. V. Thourhout, and R. Baets, "Sub-nanometer linewidth uniformity in silicon nano-photonic waveguide devices using cmos fabrication technology," IEEE J. Sel. Topics Quantum Electron., vol. 16, no. 1, pp. 316-324, Jan. 2010.

[14] F. V. Laere, G. Roelkens, M. Ayre, J. Schrauwen, D. Taillaert, D. V. Thourhout, T. F. Krauss, and R. Baets, "Compact and highly efficient grating couplers between optical fiber and nanophotonic waveguides," J. Lightw. Technol., vol. 25, no. 1, pp. 151-156, Jan. 2007.

[15] W. B. S. Pathak and D. Van Thourhout, "Design trade-offs for siliconon-insulator-based AWGs for (de)multiplexer applications," Opt. Lett., vol. 38, no. 16, pp. 2961-2964, Aug. 2013.

[16] R. Marz, Integrated Optics: Design and Modeling. Norwood, MA, USA: Artech House, 1994.

[17] F. M. V. Passaro and A. Tsarev, "Investigation of thermo-optic effect and multi-reflector tunable filter/multiplexer in SOI waveguides," Opt. Exp., vol. 13, pp. 3429-3437, 2005.

[18] A. Masood, M. Pantouvaki, G. Lepage, P. Verheyen, J. V. Campenhout, P. Absil, D. V. Thourhout, and W. Bogaerts, "Comparison of heater architectures for thermal control of silicon photonic circuits," in Proc. 10th Int. Conf. Group IV Photon., 2013, pp. 83-84.

[19] E. J. G. A. Charles and A. P. Martin, "Instrumentation for interrogating many-element fiber bragg grating arrays," Proc. SPIE, vol. 2444, pp. 257-266, 1995 .
[20] V. H. A. Ezbiri and S. E. Kanellopoulos, "High resolution instrumentation system for fibre-bragg grating aerospace sensors," Opt. Coтmun., vol. 150 , pp. $43-48,1998$.

[21] M. Fiers, T. V. Vaerenbergh, K. Caluwaerts, J. Dambre, and P. Bienstman, "Caphe: Time-domain and frequency-domain modeling of nonlinear optical components," Adv. Photon. Congr., Jun. 2012, paper IM2B.3.

[22] S. Pathak, E. Lambert, P. Dumon, D. V. Thourhout, and W. Bogaerts, "Compact SOI-based AWG with flattened spectral response using a MMI," in Proc. 8th Int. Conf. Group IV Photon., Sep. 2011, pp. 45-47.

[23] W. Bogaerts, Y. Li, S. Pathak, A. Ruocco, M. Fiers, A. Ribeiro, E. Lambert, and P. Dumon, "Integrated design for integrated photonics: From the physical to the circuit level and back," Proc. SPIE, vol. 8781, pp. 878102 1-878102-11, May 2013.

Alfonso Ruocco (S'13) received the degree in electronic engineering in 2011 from Federico II University, Naples, Italy. He joined both the Department of Information Technology, Ghent University, Ghent, Belgium, and Interuniversity Microelectronics Center, Leuven, Belgium, where he is currently working toward the Ph.D. degree. His research interests include the development of integrated spectrometers using multiplexed spectral filters. He is a Member of the IEEE Photonics Society.

Dries Van Thourhout received the degree in physical engineering and the $\mathrm{Ph} . \mathrm{D}$ degree in applied physics from Ghent University, Ghent, Belgium, in 1995 and 2000, respectively. From October 2000 to September 2002, he was with Lucent Technologies, Bell Laboratories, where he was involved in the designing, processing, and characterization of InP/InGaAsP monolithically integrated devices. In October 2002, he joined the Department of Information Technology, Ghent University, where he is currently a member of the permanent staff of the Photonics Group. He is a Lecturer or Colecturer for four courses within the Ghent University Master in Photonics Program (Microphotonics, Advanced Photonics Laboratory, Photonic Semiconductor Components and Technology). $\mathrm{He}$ is also coordinating the cleanroom activities of the research group. He is also engaged for the development of new fabrication processes for photonic devices, e.g., based on focused ion-beam etching and die-to-wafer bonding. He holds four patents. He has authored and coauthored more than 170 journal papers and invited papers at several major conferences. His research interests include the design, fabrication, and characterization of integrated photonic devices, silicon nanophotonic devices, and heterogeneous integration of InP-on-silicon. $\mathrm{He}$ holds an ERC-Starting Grant and received the 2012 Laureaat van de Vlaamse Academie van Belgie Prize. He is a Member of IEEE Photonics Society.

Wim Bogaerts (S'98-M'05) received the degree in engineering (applied physics) from Ghent University, Ghent, Belgium, in June 1998. He is currently a Professor in silicon photonics at Ghent University and IMEC. He joined the Photonics Research Group, where he started working toward the Ph.D. degree in silicon photonics. During the Ph.D. degree, he started the study on IMEC's 200-mm silicon photonics technology platform. His collaborative study with many partners evolved into the ePIXfab silicon photonics multiproject-wafer service. Since 2010, he is a Professor at Ghent University, specializing in advanced design methodologies for integrated photonics and tackling the challenges that form the design gap for silicon photonics. In June 2014, he cofounded Luceda Photonics, a Ghent University-IMEC-VUB spin-off to commercialize the IPKISS photonics design software in the rapidly growing photonic integration market. He is the coauthor of more than 150 publications in the field of silicon photonics and design methodologies, including many invitations to international conferences. He holds several patents in the field of photonics and its applications. He is a Member of the Optical Society of America and SPIE. 